\title{
Standardisation or modelling of mortality rates
}

\author{
Salvador de Mateo, Enrique Regidor
}

\begin{abstract}
Study objective - To compare the results obtained when estimating a standardised rate using the conventional technique of stratified analysis and using Poisson regression, and to evaluate the speed of the two techniques in making the calculation. Design - Cross sectional study.

Setting and participants - The trend in motor vehicle accident mortality in males from 1985 to 1992 in Spain was compared using stratified analysis and Poisson regression. In the stratified analysis the calculations were made using a specially designed spreadsheet while in the Poisson regression the statistical program used was EGRET.

Results - The stratified analysis took two hours and the Poisson regression 15 minutes to complete. In the stratified analysis a single estimate for each year was obtained, whereas the model of Poisson regression that best fitted the data included an interaction term between age and year. Conclusion - Poisson regression can be considered a serious alternative to stratified analysis when the objective is to compare mortality rates standardised by one or two variables.
\end{abstract}

\section{( $($ Epidemiol Community Health 1996;50:681-682)}

One of the most widely used procedures in comparing mortality or morbidity rates is the estimation of a summary measure standardised by age using conventional stratified analysis techniques. ${ }^{1}$ There are few statistical programs, however, that permit this type of analysis compared with the number that use log/linear Poisson modelling to estimate this measure. In this paper we compare the results obtained when estimating a standardised rate using the conventional technique of stratified analysis and using Poisson regression. We also compare the speed and ease of the two techniques in making the calculation.

\section{Methods}

We have selected as an example the trend in motor vehicle accident mortality (ICD-9,

Table 1 Poisson models for adjustment of mortality rates, variable selection and evaluation of interaction

\begin{tabular}{llrl}
\hline Model & $\begin{array}{l}\text { Deviance } \\
(d f)\end{array}$ & $\begin{array}{l}\text { Likelihood ratio } \\
\text { test }(d f)\end{array}$ & p value \\
\hline (1) Constant & $2482.72(31)$ & & \\
(2) Constant, year & $1909.34(24)$ & $573.38 \quad(7)$ & $<0.001$ \\
(3) Constant, year, age & $126.56(21)$ & $1782.78(3)$ & $<0.001$ \\
(4) Constant, year, age, year by age & - & $126.56(21)$ & $<0.001$ \\
\hline
\end{tabular}

E810-E825) in males from 1985 to 1992 in Spain. For each year we carried out stratified analysis and Poisson regression using a 486DX2 personal computer with $8 \mathrm{MB}$ of RAM.

\section{STRATIFIED ANALYSIS}

We used direct standardisation in relation to age in the stratified analysis to calculate the comparative mortality figure, taking the 1985 population as standard. We assumed the hypothesis of homogeneity of the mortality rate ratios in the different age strata. The confidence intervals were calculated after logarithmic transformation to correct for the asymmetry of the comparative mortality figure. ${ }^{1}$ All calculations were made using a specially designed spreadsheet (LOTUS).

\section{POISSON REGRESSION ANALYSIS}

In Poisson regression, where age and year of death were introduced as categorical variables, if the relative risks (RR) are constant within each age group, the regression coefficients for each year can be interpreted as the logarithms of the RR of mortality adjusted for age in respect of the base year of 1985 which was chosen as a reference. ${ }^{2}$ An analysis of possible interaction among the variables was made by means of the likelihood-ratio test or difference between deviance of hierarchical models. We show the point estimates and the confidence intervals calculated by the model using the procedure of estimated maximum likelihood. ${ }^{3}$ The statistical program used was EGRET.

\section{Results}

It took two hours to create the spreadsheet and import the data, while the Poisson regression estimate was completed in 15 minutes. The steps followed in selecting the regression model are shown in table 1 . The model that best fitted the data was the saturated model, which included an interaction term between age and year, therefore the $R R$ calculations were made with this model. In contrast with the results of the stratified analysis, in which a single estimate is obtained for each year, in statistical modelling the $R R$ rose by almost $60 \%$ in the $15-34$ year age group between 1985 and $1990(1.00 \mathrm{v}$ 1.56) whereas this increase was smaller for all other age groups (table 2).

\section{Discussion}

Although the selection of a statistical model has been deemed arbitrary, ${ }^{4}$ few choices are possible when treating a small number of variables due to the small number of models that 
Table 2 Comparative mortality figure (CMF) and relative risk (RR) point estimates in relation to mortality from motor vehicle accidents in men in Spain, 1985-92

\begin{tabular}{|c|c|c|c|c|c|}
\hline \multirow[b]{2}{*}{ Year } & \multirow{2}{*}{$\frac{\text { Stratified analysis }}{C M F(95 \% C I)}$} & \multicolumn{4}{|c|}{ Statistical modelling $R R(95 \% C I)$} \\
\hline & & Age 15-34y & Age 35-54y & Age 55-74y & Age $75+y$ \\
\hline 1985 & 1.00 & 1.00 & 1.00 & 1.00 & 1.00 \\
\hline 1986 & $1.09(1.05,1.12)$ & $1.09(1.03,1.16)$ & $1.04(0.81,1.14)$ & $1.09(0.99,1.20)$ & $1.17(1.17,1.36)$ \\
\hline 1987 & $1.17(1.14,1.20)$ & $1.26(1.19,1.34)$ & $1.09(1.00,1.19)$ & $1.06(0.97,1.17)$ & $1.17(1.17,1.36)$ \\
\hline 1988 & $1.30(1.26,1.33)$ & $1.41(1.32,1.49)$ & $1.24(1.14,1.34)$ & $1.19(1.09,1.31)$ & $1.12(1.12,1.30)$ \\
\hline 1989 & $1.43(1.39,1.46)$ & $1.57(1.48,1.66)$ & $1.36(1.26,1.48)$ & $1.28(1.17,1.40)$ & $1.19(1.19,1.38)$ \\
\hline 1990 & $1.39(1.36,1.42)$ & $1.56(1.47,1.66)$ & $1.20(1.11,1.30)$ & $1.24(1.13,1.36)$ & $1.35(1.35,1.56)$ \\
\hline 1991 & $1.33(1.29,1.36)$ & $1.54(1.46,1.63)$ & $1.17(1.08,1.27)$ & $1.13(1.03,1.24)$ & $1.10(1.10,1.28)$ \\
\hline 1992 & $1.13(1.11,1.16)$ & $1.29(1.21,1.36)$ & $1.08(0.99,1.17)$ & $0.91(0.83,1.00)$ & $0.97(0.97,1.12)$ \\
\hline
\end{tabular}

provide a good fit for the data. Thus, Poisson regression can be considered a serious alternative to stratified analysis when the objective is to compare mortality rates standardised in relation to one or two variables. Not only does the method save time, but it also solves the problem of which external standard age distribution (available from official publications) to use, since the parameters are estimated internally from the study data. With this method the choice of one population or another does not affect the size of the differences obtained with stratified analysis. Furthermore, although the small number of elements in some strata produces considerable instability, the ratio of rates obtained by Poisson regression is affected less than the summary measures obtained by stratified analysis. ${ }^{5}$ Finally, while in Poisson regression there is an evaluation of the presence of interaction, in stratified analysis it is not usual to investigate interaction between the ratio of rates in each strata and the stratification variable because the numbers are generally too small.

1 Breslow NE, Day NE. Statistical methods in cancer research. Vol II. The design and analysis of cohort studies. Lyon: IARC, 1987.

2 Frome EL, Checkoway $\mathrm{H}$. Use of Poisson regression models in estimating incidence rates and ratios. Am 7 Epidemiol in estimating inciden

3 Frome EL. The analysis of rates using Poisson regression models. Biometrics 1983;39:665-74.

4 Vandenbroucke JP. Statistical modelling: the old standardisation problem in disguise. $\mathcal{F}$ Epidemiol Community Health 1989;43:207-8.

5 Breslow NE, Day NE. Indirect standardization and multiplicative models for rates, with reference to the age adjustment of cancer incidence and relative frequency data. f Chron Dis 1975;28:289-303. 